\title{
Dynamic Response Analysis of Elastic-plastic Structures with Component Mode Synthesis
}

\author{
Peng-bo Qian \\ College of Mechanical Engineering, Nanjing University of Science and Technology, Nanjing, Jiangsu, 210094, China
}

\begin{abstract}
This paper evaluates the validity of component mode synthesis (CMS) technique for dynamic response of elastic-plastic structures. A method incorporating elastic-plastic material behaviour and the fixed interface mode synthesis technology is presented. To do this, the incremental governing equations of elastic-plastic transient problem derived from nonlinear finite element theory are linearized in each time step, the tangent stiffness matrix is used to generate the basis vector of transformation matrix. Using the proposed method, the response of a simply supported beam loaded impulsively is examined. The Numerical results indicate that the proposed method is accurate, convergent and more efficient compared with the full order finite element model.
\end{abstract}

\section{Introduction}

The dynamic analysis of elastic-plastic structure is a complicated nonlinear mechanical problem, which is typically solved using numerical method (e.g. finite element method). Because of the nonlinear constitutive relation, the time step for solving should be small enough to ensure the accuracy and reliability of solutions. If plastic deformation occurs during the time step, further iterations are required to obtain convergent results [1]. Thus, the computational cost of performing elastic-plastic dynamic response is high, especially for large or complex structures. In order to overcome this problem, the applications of reduced order models in nonlinear dynamic problems are gaining a lot of attention [2].

A commonly used technique for the dynamic responses analysis of larger or complex structures in linear system is the CMS method. This technique can reduce the size of system by dividing the structure into several subcomponents and neglecting the higher modal frequencies of subcomponents, without significantly degrading the accuracy of the solution. The CMS is originally proposed by Hurty [3], modified by Craig [4], then its theories have been further perfected, and the widespread applications are presented also. For more details, the reader can be refereed to a recent review [5].

However, the applications of CMS of linear system have been presented for many years, but of nonlinear system are still in its infancy [5]. The solutions of contact impact problem with CMS were first proposed by $\mathrm{Wu}$ and Haug [6], and then more deep studies are developed in $[7,8]$. In certain problems, the nonlinearities can be approximated as an internal force. With the help of this idea the CMS method was used to reduced modelling for geometrical nonlinear problem [9] ,material nonlinear problem[10] and some local nonlinear problem[11]. Recently, some improved CMS for nonlinear system have been a concerned topic [12].

This work is focused on investigating the validity of CMS method for dynamic analysis of elastic-plastic structures. By considering plastic behaviour of structures, one widely used CMS method named fixed-interface mode synthesis technique is enhanced. Firstly, the incremental governing equations of elastic-plastic dynamic problem are derived from nonlinear finite element theory and be linearized in each time step. Then, the tangent stiffness matrix is used to generate the basis vector of transformation matrix for fixed-interface mode synthesis method. Using the presented method, the elastic-plastic dynamic behaviours of simple beams suffered impulsive load are examined to demonstrate the validity, accuracy and efficiency of the proposed method by comparing to some published work.

\section{Formulation of CMS for elastic-plastic dynamic problem}

\subsection{Governing equations of nonlinear problem}

Limiting the motion of elastic-plastic structures in the small strain type, with the help of nonlinear finite theory, the general dynamic equilibrium equations of the problem at time can be described as [1]

$$
\mathbf{M} \ddot{\mathbf{u}}_{t}+\mathbf{F}\left(\mathbf{u}_{t}\right)=\mathbf{P}_{t}
$$

Where $\mathbf{M}, \mathbf{F}\left(\mathbf{u}_{t}\right), \mathbf{P}_{t}, \mathbf{u}_{t}$ and $\ddot{\mathbf{u}}_{t}$ are mass matrix, resisting force vector, applied force vector, nodal 
displacement vector and nodal acceleration vector of structure, respectively. The $\mathbf{F}\left(\mathbf{u}_{t}\right)$ is calculated by

$$
\mathbf{F}\left(\mathbf{u}_{t}\right)=\int_{\mathrm{V}} \mathbf{B}^{\mathrm{T}} \boldsymbol{\sigma}_{t} \mathrm{dv}
$$

Where $\mathbf{B}$ is the strain-displacement matrix of element. The $\boldsymbol{\sigma}_{t}$ stands for the stress vector at time $t$ of structure. The detailed derivation of equilibrium equation can be found in [1]. In Eq.(1), other items such as damping, can be included, but is not pertinent to the topic in this paper, so it is neglected.

After a small time interval $\Delta t$, there will be increments on system variables (e.g. stress, displacement, etc.) provided that the applied force is changed slightly at time $t+\Delta t$. And the motion of structure is still governed by Eq.(1), only by replacing variables at time $t$ with time $t+\Delta t$. That is

$$
\mathbf{M} \ddot{\mathbf{u}}_{t+\Delta t}+\mathbf{F}\left(\mathbf{u}_{t+\Delta t}\right)=\mathbf{P}_{t+\Delta t} .
$$

Subtracting Eq.(1) from Eq.(3) and considering the unbalance force in Eq.(1), this yields

$$
\mathbf{M} \Delta \ddot{\mathbf{u}}+\mathbf{F}\left(\mathbf{u}_{t+\Delta t}\right)-\mathbf{F}\left(\mathbf{u}_{t}\right)=\mathbf{P}_{t+\Delta t}-\left(\mathbf{M} \ddot{\mathbf{u}}_{t}+\mathbf{F}\left(\mathbf{u}_{t}\right)\right)=\Delta \mathbf{Q} .
$$

This equations will dominate the motion of structure during the time interval $t \sim t+\Delta t$. Typically, the resisting force vector $\mathbf{F}\left(\mathbf{u}_{t+\Delta t}\right)$ can be differentiable in the neighborhood of deformed shapes $\mathbf{u}_{t}$, so that

$$
\mathbf{F}\left(\mathbf{u}_{t+\Delta t}\right)=\mathbf{F}\left(\mathbf{u}_{t}\right)+\frac{\partial \mathbf{F}}{\partial \mathbf{u}}\left(\mathbf{u}_{t}\right) \Delta \mathbf{u}
$$

Substituting Eq.(5) into Eq.(4) and defining the tangent stiffness matrix $\frac{\partial \mathbf{F}}{\partial \mathbf{u}}\left(\mathbf{u}_{t}\right)=\mathbf{K}_{t}$, then

$$
\mathbf{M} \Delta \ddot{\mathbf{u}}+\mathbf{K}_{t} \Delta \mathbf{u}=\Delta \mathbf{Q}
$$

The Eq.(6) is the incremental governing equations of motion of elastic-plastic structures. Here, we employed the Newmark- $\beta$ method in conjunction with MNR iterative technique for solving Eq.(6). By doing so, the Eq.(6) is linearized between time steps. The tangent stiffness matrix of structure at the beginning of each time step is updated and kept unchanged in a time step. To reduce the computational effort in solution of Eq.(6), we consider using CMS method to generate a reduced order model for iterations.

\subsection{Substructuring and modal analysis}

The essence of the CMS method is the coordinate transformation, where a reduced space is generated by a set of structure modes (e.g. exact eigenmodes, static modes, interface modes, etc.) called component modes for representing the motion of structures. In this paper, we will adopt and improve fixed-interface mode synthesis approach for nonliear analysis.
The dynamic analysis of elastic-plastic structures is typically a strong nonlinear problem, and does not obey the principle of superposition. It is not possible to use CMS method directly. In order to overcome this difficult, we learn from Nickell's idea, which was originally proposed to explore the use of mode superposition method for nonlinear problems [13]. That is, the forced motion of nonlinear system over a small time interval can be represented with the nonlinear (tangent stiffness) frequency spectrum. As a result, we utilize the nonlinear frequency spectrum to generate the reduced modal space for fixed-interface mode synthesis method, and the original method is improved to be able for the nonlinear problem.

By dividing the full model into several substructures, the un-damped incremental governing equations of a substructure during the time interval $t \sim t+\Delta t$, are written in the partitioned form

$$
\begin{gathered}
{\left[\begin{array}{ll}
\mathbf{m}_{i i}^{(s)} & \mathbf{m}_{i b}^{(s)} \\
\mathbf{m}_{b i}^{(s)} & \mathbf{m}_{b b}^{(s)}
\end{array}\right]\left[\begin{array}{c}
\Delta \ddot{\mathbf{u}}_{i}^{(s)} \\
\Delta \ddot{\mathbf{u}}_{b}^{(s)}
\end{array}\right]} \\
+\left[\begin{array}{ll}
\left(\mathbf{k}_{t}^{(s)}\right)_{i i} & \left(\mathbf{k}_{t}^{(s)}\right)_{i b} \\
\left(\mathbf{k}_{t}^{(s)}\right)_{b i} & \left(\mathbf{k}_{t}^{(s)}\right)_{b b}
\end{array}\right]\left[\begin{array}{c}
\Delta \mathbf{u}_{i}^{(s)} \\
\Delta \mathbf{u}_{b}^{(s)}
\end{array}\right]=\left[\begin{array}{c}
\Delta \mathbf{q}_{i}^{(s)} \\
\Delta \mathbf{q}_{b}^{(s)}
\end{array}\right],
\end{gathered}
$$

Where the subscript $i$ indicates the interior degrees of freedom (DOFs), the subscript $b$ indicates the boundary DOFs. The superscript $(s)$ denotes the serial number of the substructure.

The fixed-interface mode synthesis approach uses two sets of substructure modes to generate transformation matrix, namely, the fixed-interface normal modes $\boldsymbol{\Phi}_{N}^{(s)}$ and constrain modes $\boldsymbol{\Phi}_{C}^{(s)}$. Fixed-interface normal modes are defined as the free vibration modes of a restrained substructure, in which all boundary degrees of freedoms are constrained and the displacement are set to zero, i.e., $\Delta \mathbf{u}_{b}^{(s)}=\mathbf{0}$. Thus, the Eq.(7) is reduced to an eigenvalue problem

$$
\left(\left(\mathbf{k}_{t}^{(s)}\right)_{i i}-w^{2} \mathbf{m}_{i i}^{(s)}\right) \boldsymbol{\Phi}_{i i}^{(s)}=\mathbf{0}
$$

The constraint modes are the static deflections of substructure that result due to successive unit displacements at each boundary degrees of freedoms, all other boundary degrees of freedoms being totally constrained. Thus, the constraint modes of substructure can be calculated by as

$$
\boldsymbol{\Phi}_{C}^{(s)}=\left[\begin{array}{l}
\boldsymbol{\Psi}_{i b}^{(s)} \\
\mathbf{I}_{b b}^{(s)}
\end{array}\right]=\left[\begin{array}{c}
-\left(\left(\mathbf{k}_{t}^{(s)}\right)_{i i}\right)^{-1} \mathbf{k}_{i b}^{(s)} \\
\mathbf{I}_{b b}^{(s)}
\end{array}\right]
$$

Remaining the lowest fixed-interface normal modes $\boldsymbol{\Phi}_{i k}^{(s)}$, the coordinate transformation of substructure can be written as

$$
\Delta \mathbf{u}^{(s)}=\left[\begin{array}{cc}
\boldsymbol{\Phi}_{i k}^{(s)} & \boldsymbol{\Psi}_{i b}^{(s)} \\
\mathbf{0} & \mathbf{I}_{b b}^{(s)}
\end{array}\right]\left[\begin{array}{c}
\Delta \mathbf{a}_{i}^{(s)} \\
\Delta \mathbf{u}_{b}^{(s)}
\end{array}\right]
$$


Where $\Delta \mathbf{a}^{(s)}$ is denoted as the modal displacement vector. The total number of which is equal to the number of retained fixed-interface modes plus the number of boundary DOFs.

\subsection{Synthesis of whole system}

Finally, all the substructures are assembled to form the full system expressed by modal coordinates, which has a reduced order of DOFs. The process of assembling is similar to finite element analysis. With the consideration of displacement compatibility and force equilibrium of the interface DOFs at the conjunction region of substructures, and eliminating repeated interface DOFs, the dynamic equilibrium equations of full system can be expressed as follows

$$
\overline{\mathbf{M}} \Delta \ddot{\mathbf{a}}+\overline{\mathbf{K}}_{t} \Delta \mathbf{a}=\boldsymbol{\Phi}^{\mathrm{T}} \Delta \mathbf{P}=\Delta \mathbf{Q}
$$

Where $\boldsymbol{\Phi}$ is the modal matrix of full system assembled from all substructures. The $\overline{\mathbf{M}}=\boldsymbol{\Phi}^{\mathrm{T}} \mathbf{M} \boldsymbol{\Phi}, \overline{\mathbf{K}}_{t}=\boldsymbol{\Phi}^{\mathrm{T}} \mathbf{K}_{t} \boldsymbol{\Phi}$ and $\Delta \mathbf{Q}=\boldsymbol{\Phi}^{\mathrm{T}} \Delta \mathbf{P}$ are modal mass matrix, modal tangent stiffness matrix and modal external force vector of full system, respectively.

\section{Numerical example}

Considering a simply supported beam under distributed load $p(t)$ as depicted in Fig. 1. This problem has been investigated by many researchers [14-15].The dimensions of the beam are shown as follow: $L=0.762 \mathrm{~m}, h=0.0508$ $\mathrm{m}, w=0.0254 \mathrm{~m}$. The material of this beam is assumed to be ideal plastic with $\mathrm{E}=206.8 \mathrm{GPa}, \sigma_{y}=344.74 \mathrm{MPa}, \rho=$ $7800 \mathrm{Kg} / \mathrm{m}^{3}$.
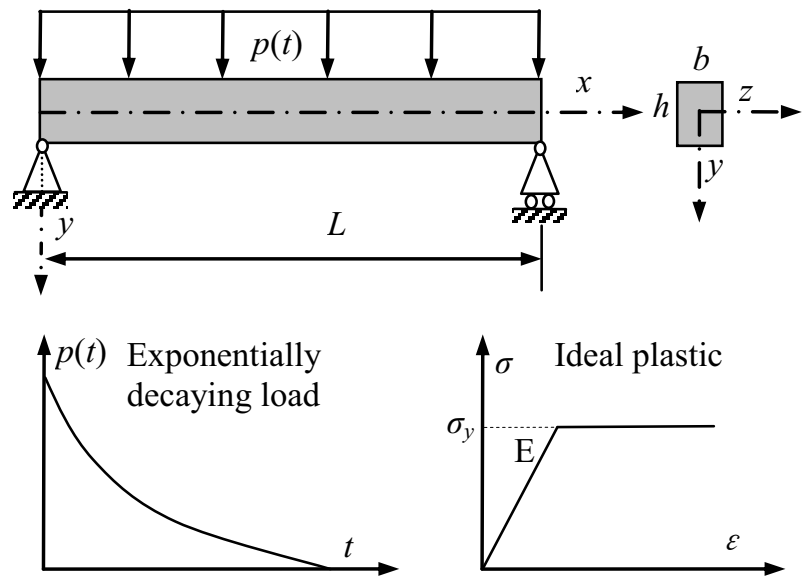

Figure 1. Simply supported beam system

The time variation of the exponentially decaying load is given as $p(t)=n p_{0} \mathrm{e}^{-2 t / \mathrm{T}}$. where $p_{0}$ is static collapse load defined as the load causing a plastic hinge to occur at the midpoint of the beam. Then $n=2.0$ and $\mathrm{T}$ is the fundamental time period of the beam. The time step of this example equals $1.0 \mathrm{e}^{-5} \mathrm{sec}$.

\subsection{Convergence and accuracy study}

First, a convergence study was developed based on the peak deflection of midpoint of the beam for exponentially decaying load using the proposed method. With the uniform division, each substructure along the beam is discretized into 2 Euler beam elements, and the fixedinterface normal modes of substructure retains 1 . The convergent plot of substructures is depicted in Figure 2. The first peak value of midpoint non-dimensional deflection is 8.3 reported in reference [14]. The nondimensional deflection $\delta$ is obtained by normalizing the deflection with respect to $\Delta$, the static elastic deflection caused by the load $p_{0}$. As the number of substructures is increased, the accuracy of the solution for the midpoint deflection is improved obviously as shown in Figure 2.

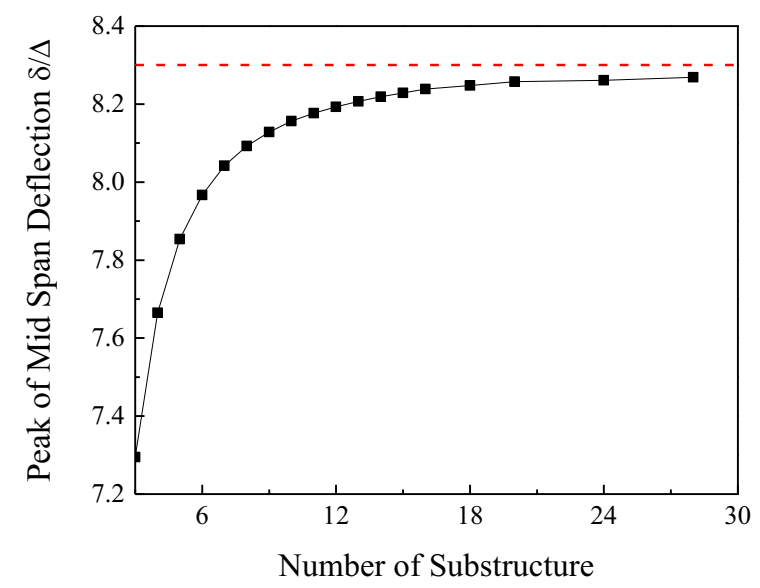

Figure 2. Convergence study of CMS method

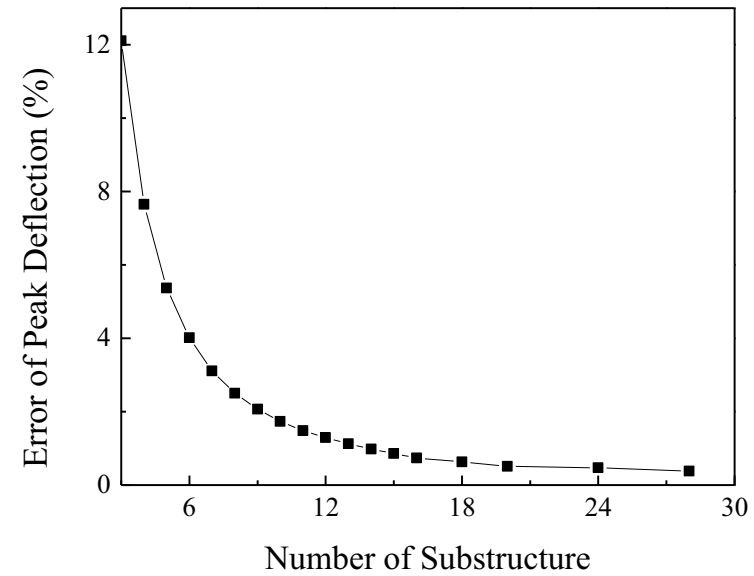

Figure 3. Relative error of the CMS method

The relative error of the solution is calculated by $\left(\delta_{\mathrm{CMS}}-\delta_{\text {Ref }}\right) / \delta_{\text {Ref }}$, where $\delta_{\mathrm{CMS}}$ is the numerical solution of the first peak value of deflection from the proposed method, $\delta_{\text {Ref }}$ is the first value of deflection reported in reference [14]. The relationship between relative error and substructure number is shown in Figure 3. It gives the information that the relative error is decreased from 14 percent with 2 substructures to 2 percent with 8 substructures rapidly, at the begining of the curve. After that, with the increase in the number of substructures, the 
relative error is reduced significantly slow. For instance, the relative error is 0.378 percent when the substructure number is increased to 28 . The Figure 3 also indicates that a solution with $<2 \%$ relative error can be obtained at the division of 10 substructures.

Then, for the succeeding simulations in this example, the division strategy of 10 substructures with 1 fixedinterface normal modes will be used always. The time history of midpoint deflection of the beam subjected to exponentially decaying load is compared with previous published work in Figure 4. The comparisons show that the result of the proposed method agree well with those of Baron [14], and Nagarajan [15].

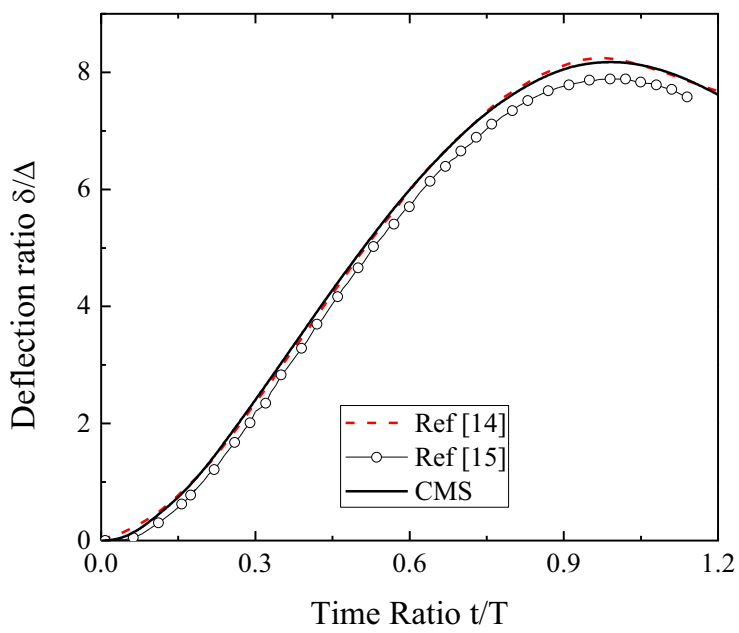

Figure 4. Deflection response of the simply supported beam

\subsection{Computational efficiency study}

For a concrete elastic-plastic dynamic analysis, it is obviuous that the description with Eq.(11) has much smaller size than the description with Eq.(6). Nevertheless, an additional coordinate transformation is needed to generated Eq.(11) compared with Eq.(6). The time cost of elastic-plastic dynamic analysis should include two parts, the time for solving governing equations and the time for generating governing equations. In order to evaluate the efficiency of the proposed method, we record the time cost of simulating this example with the proposed method and with full order model (description with Eq.(6)), respectively. Although, this example is a simple beam structure, the result still can explain some characteristics of the proposed method.

Table 1. Time cost of simulation.

\begin{tabular}{|l|l|l|l|}
\hline Method & Discretization & Time cost (s) & Time ratio \\
\hline Full model & $N_{e}=20$ & 10.72 & 1.00 \\
\hline CMS & $N_{s}=10$ & 7.15 & 0.66 \\
\hline
\end{tabular}

The central processing unit (CPU) time for simulation and obtained benefit in different analysis are listed in Table 1. It is clear that the CMS method has higher computational efficiency than full order model. For this example, the computational time cost of proposed method is only about 0.66 times as the full order model.

\section{Conclusions}

In order to evaluate the validity of CMS for dynamic responses of elastic-plastic structures, a numerical method incorporating elastic-plastic theory and fixedinterface component mode synthesis technique is proposed. To do this, the incrementally governing equations of elastic-plastic dynamic problem are linearized over a small time step, the tangent stiffness matrix is used to generate the transformation matrix. The presented method is demonstrated by the analysis of dynamic elastic-plastic response of simply supported beams. The material of beam is assumed to be ideal plastic and the load is exponentially decaying. Numerical results are in excellent agreement with those obtained by previous researchers. The comparison of the computational time for example also demonstrates that the proposed method has higher computation efficiency compared with finite element method.

\section{Acknowledgment}

This research has been supported by Postdoctoral Fund of JiangSu (1402007B), the support is gratefully acknowledged.

\section{References}

1. O. C. Zienkiewicz, R.L.T, The Finite Element Method for Solid and Structural Mechanics, (2006).

2. A. Corigliano, M. Dossi, S. Mariani, COMPUT METHOD APPL M, 290, 127-155, (2015).

3. W. C. Hurty, AIAA J, 3 678-685. (1965).

4. R. R. Craig, M. C. C Bampton, AIAA J, 6, 13131319. (1968)

5. D.d Klerk, D. Rixen, S. Voormeeren, AIAA J, 46, 1169-1181, (2008).

6. S. C. Wu, E. J. Haug, J MECH DESIGN 112, 390398, (1990).

7. A. Guo, S. Batzer, J VIB ACOUST 126, 126-131, (2004)

8. Y, Shen, X. Yin, APPL MATH MODEL, 40, 19721988, (2016).

9. R. J. Kuether, M. S. Allen, Dynamics of Coupled Structures, 1, 167-178, (2014).

10. J. Bond, T. Khraishi, INT J MECH MATER DES, 5, 365-380, (2009).

11. D-K, Kim, J-S. Bae, I. Lee, J-H. Han, J SPACECRAFT ROCKETS, 42, 66-77. (2005)

12. I. P. Krishna, C. Padmanabhan, NONLINEAR DYNAM, 63, 561-586. (2011)

13. R. E. Nickell, COMPUT METHOD APPL M, 7, 107-129. (1976)

14. M. L. BARON, H. H. B, P. WEIDLINGER. J ENG $\mathrm{MECH}, \mathbf{8 9}, 23-42$. (1961)

15. S. Nagarajan, E. P. Popov, COMPUT STRUCT, 4, 1117-1134, (1974) 